This item was submitted to Loughborough's Research Repository by the author.

Items in Figshare are protected by copyright, with all rights reserved, unless otherwise indicated.

\title{
Calculations of explosion deflagrating flames using a dynamic flame surface density model
}

PLEASE CITE THE PUBLISHED VERSION

PUBLISHER

(C) Elsevier

VERSION

AM (Accepted Manuscript)

LICENCE

CC BY-NC-ND 4.0

\section{REPOSITORY RECORD}

Ibrahim, Salah S., Sreenivasa Rao Gubba, Assaad R. Masri, and W. Malalasekera. 2019. "Calculations of Explosion Deflagrating Flames Using a Dynamic Flame Surface Density Model”. figshare. https://hdl.handle.net/2134/5566. 
This item was submitted to Loughborough's Institutional Repository (https://dspace.lboro.ac.uk/) by the author and is made available under the following Creative Commons Licence conditions.

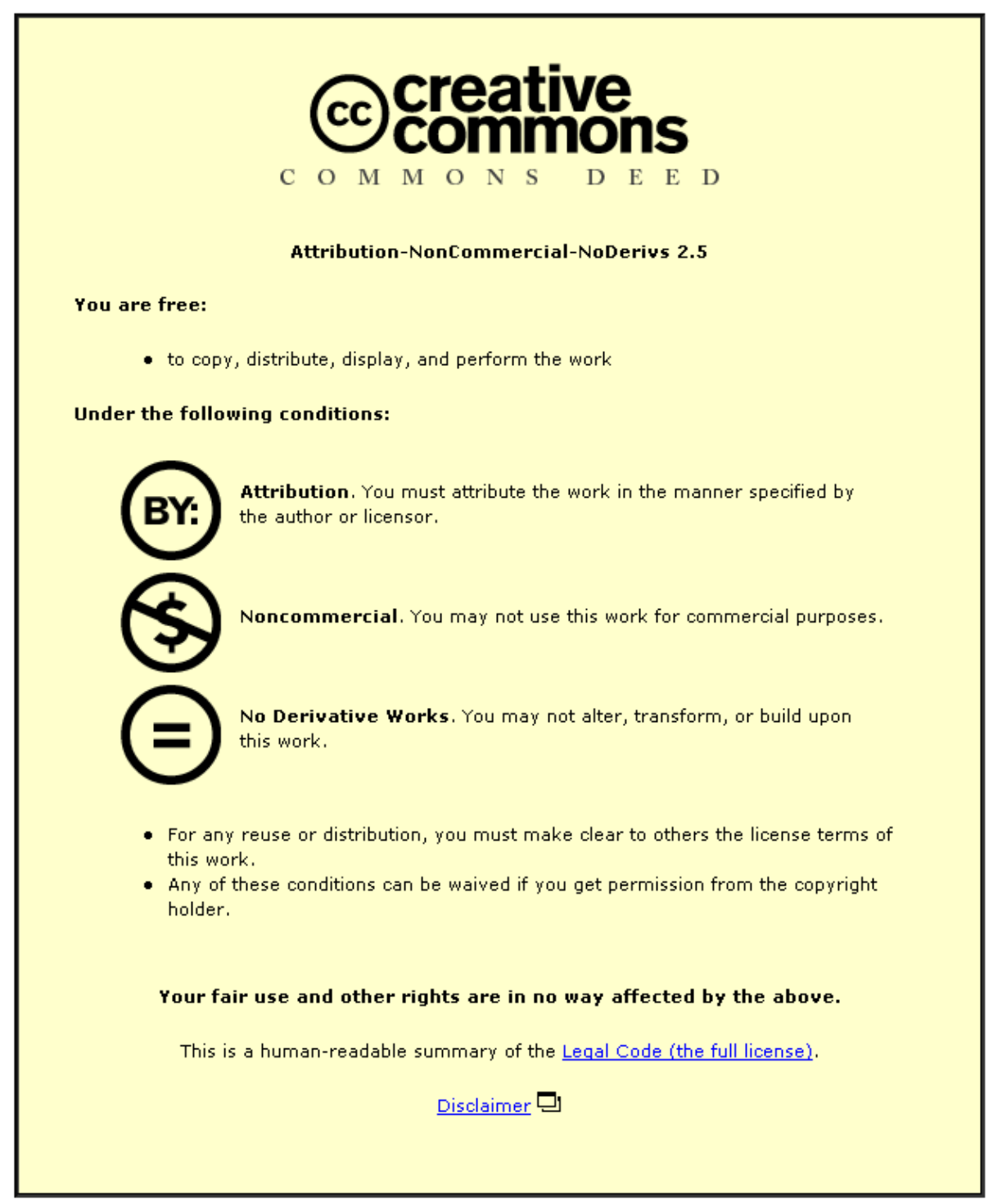

For the full text of this licence, please go to: http://creativecommons.org/licenses/by-nc-nd/2.5/ 
Journal of Loss Prevention in the Process Industries "Special issue on CFD Simulations for Explosion Phenomena"

\title{
Calculations of Explosion Deflagrating Flames using a Dynamic Flame surface density Model
}

S. S. Ibrahim ${ }^{1 *}$ S. R. Gubba ${ }^{2}$, A. R. Masri ${ }^{3}$ and W. Malalasekera ${ }^{2}$

\author{
${ }^{1}$ Aeronautical and Automotive Engineering \\ Loughborough University, Loughborough, LE11 3TU, UK \\ ${ }^{2}$ Wolfson School of Mechanical and Manufacturing Engineering \\ Loughborough University, Loughborough, LE11 3TU, UK \\ ${ }^{3}$ School of Aerospace, Mechanical and Mechatronic Engineering \\ The University of Sydney, Sydney, NSW 2006, Australia
}

*Corresponding author: Dr. Salah S Ibrahim

Aeronautical and Automotive Engineering Loughborough University

Loughborough, LE11 3TU, UK

Tel: +441509227253

Fax: +44 1509227275

E-mail: S.S.Ibrahim@Lboro.ac.uk 


\title{
Calculations of Explosion Deflagrating Flames using a Dynamic Flame Surface Density Model
}

\begin{abstract}
Explosion deflagrating flames in a small scale vented chamber, with repeated obstacles are simulated using the large eddy simulation (LES) technique for turbulent reacting flows. A novel dynamic flame surface density (DFSD) combustion model, based on the laminar flamelet concept has been used to account for the mean chemical reaction rate. All cases considered here start with a stagnant, stoichiometric propane/air mixture. Three configurations with two baffle plates and a solid square obstacle, at different axial locations from the bottom ignition centre are examined. Numerical calculations of explosion generated pressure histories; flame characteristics such as structure, position, speed and acceleration are validated against published experimental data. Influence of the relative position of baffles plates with respect to the origin of the ignition are examined and discussed. Qualitative comparisons of the computed reaction rate are also made with images of Laser Induced Fluorescence from $\mathrm{OH}$ measurements. Good agreement obtained between numerical predictions and experimental measurements confirms the applicability of the newly developed dynamic model to predict the dynamics of explosion deflagrating flames.
\end{abstract}

Keywords: Explosion modelling, LES, Deflagrating flames, Flame surface density.

\section{Introduction}

Computational Fluid Dynamics (CFD) models are able to predict the transient nature of explosion deflagrating flames, which plays pivotal role in the safe design of on- and off-shore chemical processing plants. In accidental explosions situations, the damage caused by the explosion generated over pressure due to flame acceleration and its interactions with the presented solid obstacles is generally enormous. Several experimental and numerical studies focus on the influence of the shape and size of the solid obstacles and their interactions with propagating flame, in generating overpressure (e.g. Ibrahim \& Masri, 2001; Ibrahim, Hargrave, \& Williams, 2001; Patel, Jarvis, Ibrahim, \& Hargrave, 2002). However, uncertainties remain on the appropriate mathematical description of the interactions between the propagating flame and the turbulent flow formed within solid boundaries (Chakravarthy, Smith, \& Menon, 1999).

Flamelet modelling of premixed, turbulent deflagrating flames, provides a mean to introduce chemical and turbulence time scales by considering a thin laminar flame in a turbulent flow field. The key goal behind flamelet modelling is to incorporate various flamelet stretching mechanisms to account for effective turbulence time scales. Much of the flamelet modelling literature focuses on deriving effective turbulent burning velocity (Abdel-Gayed, Bradley, \& Lawes, 1987). The alternative modelling strategy has been pursued for the flamelet regime since the first introduction of the Bray-Moss-Libby (BML) model (Bray, Libby, \& Moss, 1985). This approach is based on evaluating the flamelet surface area to volume ratio (flamelet surface density), which can be computed via an algebraic (Bray, 1990) or through a transport equation. Recently, the transport equation for the flamelet surface density has been the subject of many works (Hawkes 
\& Cant, 2001; Patel, Ibrahim, Yehia, \& Hargrave, 2003), and is an attempt to handle extreme cases when the coupling between the flow-field and flame front is intense.

In this paper a dynamic CFD model based on the Large Eddy Simulation (LES) technique is used to calculate explosion deflagrating flames in a vented explosion chamber. The combustion model is based on dynamic determination of the resolved and unresolved flame surface density (FSD), which allows for the sub-grid scale (SGS) dynamic effects of the local flame interactions. The main objective of the present work is two folded. Firstly, to validate the applicability of the developed CFD-LES technique predicting flame propagation inside semi-confined explosion chamber. Secondly, to study the influence of position of the baffles inside vented chamber with respect to the ignition origin.

Results are presented for three different flow configurations involving mainly different solid obstruction arrangements inside the explosion chamber. The predicted results are validated against experimental data recently published by the combustion group at The University of Sydney (Kent, Masri, \& Starner, 2005). Model validation is carried out in terms of the explosion generated pressure, the speed and acceleration of the deflagrating flame as well as images of laser induced fluorescence (LIF) from $\mathrm{OH}$ measurements collected as the flame is propagating near the final obstacle. The developed dynamic model found to give very good predictions for the flame characteristics at different times following ignition.

\section{The Test Case}

The experimental test cases used in the present investigation to validate the LES predictions of explosion deflagrating flames are those, reported by The University of Sydney combustion group (Kent et al., 2005). A schematic diagram of the laboratory scale explosion rig, with possible baffle plates and a solid square obstacle is illustrated in Fig. 1. The chamber is of $50 \mathrm{~mm}$ square cross section with a length of $250 \mathrm{~mm}$ and having a total volume of $0.625 \mathrm{~L}$. This chamber is of particular interest because of its smaller volume and its capability to hold a deflagrating flame in strong turbulent environment, generated due to the presence of solid obstacles at different downstream locations from the bottom ignition end. This chamber can accommodate a maximum of three baffle plates at various baffle stations and a square solid obstacle in the path of the deflagrating flame. These baffle stations are named as S1, S2 and S3 and located at 20, 50 and $80 \mathrm{~mm}$ respectively from the ignition point as shown in Fig. 1. Each baffle plate is of $50 \times 50 \mathrm{~mm}$, aluminium frame, constructed from $3 \mathrm{~mm}$ thick sheet, consisting of five $4 \mathrm{~mm}$ wide bars each with a $5 \mathrm{~mm}$ wide space spreading them through out the chamber. A solid square obstacle of $12 \mathrm{~mm}$ cross-section is centrally located at $96 \mathrm{~mm}$ from the bottom ignition end of the chamber.

For the present investigation, three configurations having two baffle plates, at different stations, shown in Fig. 2 are considered. All the baffle plates are aligned at 90 degrees to the solid obstacle in the chamber. A Piezo-resistive pressure transducer with a range of $0-1 \mathrm{bar}$ and a response time of $0.1 \mathrm{~ms}$ is used to measure the overpressure in the explosion chamber. The pressure transducer is positioned at the ignition end of the vessel. Experimental measurements recently published by Kent et. al. (2005) are used here for validation of CFD simulations. 
Images of Laser-Induced Fluorescence from the hydroxyl radical, $\mathrm{OH}$ are also collected as the flame is propagating around the final obstacle, which has a square cross section. A Quanta-Ray PDL-3 Pulsed Dye Laser manufactured by Spectra-Physics was pulsed through a dye mixture to produce a $566 \mathrm{~nm}$ visible yellow burst, which is then frequency doubled to the required $283.9 \mathrm{~nm}$. The fluorescence from $\mathrm{OH}$ is collected at 90 degrees using a Photometrics SenSys CCD camera and an interference filter to ensure that only ultra-violet light of wavelength $313 \mathrm{~nm}$ in allowed to propagate. The monitored LIF-OH region covers an area of approximately $15 \times 28 \mathrm{~mm}$, which extends from the side of the square obstacles to a few millimeters downstream.

\section{Mathematical and Numerical Modelling}

Transient calculations of explosion deflagrating flames in vented chambers shown in Fig. 2, are carried out using large eddy simulation (LES) methodology. In LES, large eddies above a cut-off length scale are resolved and the small ones are modelled by assuming isotropic in nature, using sub-grid scale (SGS) models. Favre filtered (density weighted) conservation equations of mass, momentum, energy and reaction progress variable are solved together with state equation. Turbulence is modelled using the classical Smagorinsky model (Smagorinsky, 1963) and the model coefficient is calculated from instantaneous flow conditions using the dynamic determination procedure developed (Moin, Squires, Cabot, \& Lee, 1991) for compressible flows.

Modelling the mean chemical reaction rate in deflagrating flames is very challenging due to its non-linear relation with chemical and thermodynamic states, and often characterized by propagating thin reaction layers thinner than the smallest turbulent scales. The major difficulty in the modelling of reaction rate is due to sharp variation of thermo chemical variables through the laminar flame profile, which is typically very thin (Veynante \& Poinsot, 1997). This issue is strongly affected by turbulence, which causes flame wrinkling and thereby forming the most complex three way thermo-chemical-turbulence interactions. However, assuming the single step irreversible chemistry and the Zeldovich instability (thermal diffusion), i.e. unit Lewis number will reduce the complexity of the whole system. The chemical status is described by defining the reaction progress variable $c$ from zero to one in unburned mixture and products respectively, based on fuel mass fraction. Mathematically it can be derived as, $1-Y_{f u} / Y_{f u}^{0}$. Here $Y_{f u}$ is the local fuel mass fraction and $Y_{f u}^{0}$ is fuel mass fraction in unburned mixture.

The mean SGS chemical reaction rate $\overline{\dot{\omega}}_{c}$ in reaction progress variable equation (not shown here) is modelled by following the laminar flamelet approach as:

$$
\overline{\dot{\omega}}_{c}=\rho_{u} u_{L} \bar{\Sigma}
$$

where $\rho_{u}$ is the density of unburned mixture, $u_{L}$ is the laminar burning velocity, and $\bar{\Sigma}$ is the flame surface density. Following recent success (Gubba, Ibrahim, Malalasekera, \& Masri, 2007) in accounting the unresolved chemical reaction rate, in addition to the mean chemical reaction rate by using novel dynamic flame surface density (DFSD) model (Knikker, Veynante, \& Meneveau, 2004), present work is carried out using 
DFSD model shown in equation (2). The term, mean filtered flame surface density $\bar{\Sigma}=\overline{|\nabla c|}$ (in Eq. 1), can be split into two terms as resolved and unresolved:

$$
\bar{\Sigma}=\overline{|\nabla c|}=\underbrace{\prod(\bar{c}, \bar{\Delta})}_{\text {Resolved }}+\underbrace{f(\bar{c}, \bar{\Delta}, \Pi(\bar{c}, \bar{\Delta}))}_{\text {Unresolved }}
$$

where $c$ is the mean reaction progress variable, $\bar{\Delta}$ is the filter width and $C s$ is the model coefficient. An over-bar describes application of the spatial filter, while the hat ( $)$ denotes test filter application. The resolved term in the above equation is evaluated using the expression given by (Knikker et al., 2004) and the unresolved term is calculated as:

$$
\lambda=\bar{\Sigma}-\Pi(\bar{c}, \bar{\Delta})=\overline{|\nabla c|}-\Pi(\bar{c}, \bar{\Delta})
$$

Defining $\gamma$ as a ratio of test filter to grid filter, i.e. $\langle\Delta\rangle / \bar{\Delta}$, such that the test filter $\langle\Delta\rangle$ is greater than the grid filter $\bar{\Delta}$. Applying the test filter to flame surface density (Eq. 2 ) leads to:

$$
\langle\bar{\Sigma}\rangle=\langle\overline{|\nabla c|}\rangle=\underbrace{\prod(\langle\bar{c}\rangle,\langle\bar{\Delta}\rangle)}_{\text {Resolved@testfilter }}+\underbrace{[\langle\overline{\nabla c \mid}\rangle-\Pi(\langle\bar{c}\rangle,\langle\bar{\Delta}\rangle)]}_{\text {Unresolved@testfilter }}
$$

From the above equation, unresolved flame surface density contribution at the test filter level can be written as

$$
\Lambda=[\langle\overline{|\nabla c|}\rangle-\Pi(\langle\bar{c}\rangle,\langle\bar{\Delta}\rangle)]
$$

Assuming the sub-grid scale contribution of unresolved flame surface density at test filter is the same as that at grid filter and relating $\lambda$ and $\Lambda$ by using Germano identity (Germano, Piomelli, Moin, \& Cabot, 1991):

$$
\begin{aligned}
\Lambda-\langle\lambda\rangle= & {[\langle\overline{|\nabla c|}\rangle-\Pi(\langle\bar{c}\rangle,\langle\bar{\Delta}\rangle)]-[\langle\overline{|\nabla c|\rangle}-\langle\Pi(\bar{c}, \bar{\Delta})\rangle]} \\
& \Lambda-\langle\lambda\rangle=[\langle\Pi(\bar{c}, \bar{\Delta})\rangle-\Pi(\langle\bar{c}\rangle,\langle\bar{\Delta}\rangle)]
\end{aligned}
$$

The sub-grid scale flame surface density contribution from the above equation can be added to the resolved flame surface density (Eq. 4) with a model coefficient $C s$ in order to obtain total flame surface density. Hence the flame surface density can be expressed as:

$$
\bar{\Sigma}=\prod(\bar{c}, \bar{\Delta})+\operatorname{Cs}\left[\left\langle\prod(\bar{c}, \bar{\Delta})\right\rangle-\Pi(\langle\bar{c}\rangle,\langle\bar{\Delta}\rangle)\right]
$$

The model coefficient $C s$ in above equation is dynamically obtained by identifying subgrid scale flame surface as a fractal surface (Knikker et al., 2004) as follows:

$$
C s=\frac{1}{1-\gamma^{2-D}}\left[\left(\frac{\bar{\Delta}}{\delta_{c}}\right)^{D-2}-1\right]
$$

where $\delta_{c}$ is the lower cut-off scale, $\gamma$ is the ratio of test filter to grid filter and $D$ is the fractal dimension, calculated dynamically (Knikker et al., 2004).

\subsection{LES simulations}

Favre-filtered conservation equations are computationally solved using a compressible, in-house LES code, PUFFIN (Kirkpatrick, Armfield, Masri, \& Ibrahim, 2003), which can simulate various industrial flow problems. Conservation equations are implicitly filtered using box filter and then discretized using the finite volume method. The discretization is based on control volume formulation on a staggered, non-uniform, Cartesian grid. A second order central difference approximation is used for diffusion, 
advection and pressure gradient terms in the momentum equations and for gradient terms in the pressure correction equation. Conservation equations for scalars use second order central difference scheme for diffusion terms. The third order upwind scheme of Leonard, QUICK (Leonard, 1979) and SHARP (Leonard, 1987) is used for advection terms of the scalar equations to avoid problems associated with oscillations in the solution. The QUICK scheme is also sometimes used for the momentum equations in areas of the domain where the grid is expanded and accurate calculation of the flow is less important. The equations are advanced in time using fractional step method. CrankNicolson scheme is used for the time integration of momentum and scalar equations. A number of iterations are required at every time step due to strong coupling of equations with one other.

Solid boundary conditions are applied at the bottom, vertical walls, for baffles and obstacle by setting the normal and tangential velocity components to zero, which ideally represents impermeable and no-slip conditions. The walls and obstacles are isothermal and same temperature is maintained through out the simulations. The wall shear is calculated by the $1 / 7^{\text {th }}$ power-law wall function of Werner and Wengle (Werner $\&$ Wengle, 1991), taking the form of $\tau_{w}=W(\tilde{u}, y)$, where $\tau_{w}$ is the wall shear stress, $W$ is a functional dependence, $y$ is the distance of the grid point form the wall and $\tilde{u}$ is the tangential velocity at $y$. Outflow boundary conditions are used at the open end of combustion chamber. A non-reflecting boundary condition (Kirkpatrick et al., 2003), analogous to commonly used convective boundary condition, in incompressible LES is used to prevent reflection of pressure waves at this boundary. The initial conditions are quiescent with zero velocity and reaction progress variable. Ignition is modelled by setting the reaction progress variable to 0.5 with in the radius of $4 \mathrm{~mm}$ at the bottom centre of chamber.

The governing equations, discretized by the finite volume method, are solved using a Bi-Conjugate Gradient solver with an MSI pre-conditioner for the momentum, scalar and pressure correction equations. The time step is limited to ensure the CFL number remains less than 0.5 with an extra condition that the upper limit for $\delta t$ is $0.3 \mathrm{~ms}$. The solution for each time step requires around 8 iterations to converge, with residuals for the momentum equations less than $2.5 \mathrm{e}-5$ and scalar equations less than $2.0 \mathrm{e}-3$. The mass conservation error is less than $5.0 \mathrm{e}-8$.

\subsection{Computational Domain}

The computational domain has dimensions of $50 \times 50 \times 250 \mathrm{~mm}$, where the explosion deflagrating flame takes place over baffles and solid obstacles. This domain is adequately extended to $325 \mathrm{~mm}$ in $x, y$ and $250 \mathrm{~mm}$ in $z$ direction with far-field boundary conditions. LES simulations are carried out for 3-D, non-uniform, Cartesian co-ordinate system for a compressible flow, having low Mach number. LES simulations are performed for a stagnant propane/air mixture having equivalence ratio 1.0 in the explosion chamber with a grid resolution of $90 \times 90 \times 336$ (2.7 million).

\section{Results and Discussion}

Results from the LES simulations of stagnant, stoichiometric propane/air explosion deflagrating flames over repeated solid obstacles are presented and discussed in this section. A novel DFSD model (Gubba et al., 2007; Knikker et al., 2004), to 
account the SGS chemical reaction rate is used to model explosions in the vented chambers shown in Fig. 2. Three flow configurations with two baffle plates at different stations and a solid square obstacle at a fixed position as shown in Fig. 2 are considered for the present study. The baffle plates and solid square obstacle are aimed to generate turbulence by disrupting the flame propagation with a blockage ratio of $40 \%$ (for each baffle plate) and $25 \%$ respectively. Primary objective of the present work is to validate the application of DFSD model in predicting vented explosions dynamics. Secondly, influence of the position of individual baffle plate in generating overpressure, due to the interactions with deflagrating flames, with respect to the origin of ignition is examined.

Figure 3 presents the predicted flame structure using reaction rate contours at various instants of time after ignition. It is identified in all these configurations that, following ignition, flame front expanded hemi-spherically with a velocity of $\Theta \mathrm{u}_{L}(\Theta$ is the thermal expansion factor) in axial direction until it reaches first baffle plate and flame skirt elongates with laminar burning velocity, $u_{L}$ in radial direction. The time taken by the flame front to reach solid square obstacle, to generate maximum overpressure and venting of the flame were strongly influence by this initial laminar behaviour of the flame. Typically, five identical instants i.e. at 6, 8, 10, 11.5 and 12.0 $\mathrm{ms}$ after ignition are considered from all these configurations. At these instances, turbulent fluctuations are computed at the leading edge of propagating flame. This facilitates to analyse the flame position, behaviour and its structure at any chosen time.

For example at $6 \mathrm{~ms}$, the flame is jetting out of the baffle plate at $\mathrm{S} 1$ in configuration 2 and 3, with similar flame structure. The RMS of axial velocity is computed as $2 \mathrm{~m} / \mathrm{s}$ for both these configurations at $6 \mathrm{~ms}$. However, the flame is found to be hemi-spherical and laminar with a negligible RMS fluctuations $(<0.2 \mathrm{~m} / \mathrm{s})$ in configuration 1 at $6 \mathrm{~ms}$. Similarly, considering the reaction rate contours at $10 \mathrm{~ms}$, the flame is about to interact with baffle plate at S3 with totally different flame structure and respective RMS fluctuations of 4 and $5 \mathrm{~m} / \mathrm{s}$ from configuration 1 and 2 . The flame in configuration 3 found to be more turbulent at $10 \mathrm{~ms}$ with RMS velocity of $8 \mathrm{~m} / \mathrm{s}$ (at its peak in this configuration) and about to interact with solid square obstacle. Hence, the differences in flame position, flame front structure and the degree of wrinkles are found to be directly related to the axial location of baffles with respect to the origin of ignition.

Figure 4 shows characteristic comparison of overpressure histories for three configurations, from experimental measurements and LES simulations. Due to the blockage of flow and interactions of flame by/with second baffle plate in configuration 2 and 3, a small hump in pressure history is noticed at around $8 \mathrm{~ms}$. It is clear that the rate of pressure rise and its trend including first hump are predicted well except for configuration 3 where the computed rate of increase of pressure is slower than measurements indicating a faster decay of turbulence between the second baffle plate and the square obstacle. It is also worth noting here that the pressures reported here are measured close to the chamber's plane and these may be different if measured at the base plate near the ignition source due to a possible pressure gradient within this chamber. From the experimental measurements, the overpressure is found to be oscillating after the peak overpressure, while burning the remaining trapped mixture after blow down of the main flame. It has been found in our preliminary studies, that the DFSD model is able predict the oscillating behaviour of the overpressure while burning the trapped fuel/air mixture. However, this is not verified for any of the configurations 
presented in this paper for two reasons. The first one is, as this report is interested in flame-obstacle interactions, no point in carrying simulations once flame left the chamber. The second is, cost of the computational time as it generally takes 32 days of a Viglen Genie computer having a Xeon ${ }^{\circledR}$ processor, with 3 GB RAM for 13 ms LES solution.

A comparison of the flame position from experiments and LES predictions is shown in Fig. 5. In case of experiments, the flame position is extracted from high speed video images by locating the farthest location of the flame front from ignition bottom end. From LES calculations, the flame position is obtained by locating the farthest location of the leading edge of the flame front from the bottom end (defined here as the most down stream location of the flame, where $c=0.5$ from the ignition point). While results for configuration 2 almost fully overlap, a slightly faster rate of propagation across the chamber is computed for configurations 1 and 3. This is evident only in the last few milliseconds of propagation where the flame is experiencing the highest levels of turbulence.

Figure 6 shows comparison between experimental measurements and numerical predictions of flame speed and acceleration. Also the position of baffle plates and the solid square obstacle are shown in Fig. 6, to identify the influence of the obstacles. Flame speed is calculated from the rate of change over successive images in case of experiments and as a first derivative of the flame position with respect to time in LES. It should be noted here that in case of experimental measurements there is $2000 \mathrm{fps}$ limitation on the high-speed digital camera, which eventually controls the resolution of the measurements. Due to this limitation, the drop in flame speed after the square obstacle is not captured correctly and however, predictions from LES are more continuous. For clarity, experimental measurements are represented by square symbols in Figure 6(a) and (b). At the location of the square obstacle, the highest flame speed is obtained for configuration 1 and this location also corresponds to the highest flame acceleration. It is interesting to note in configuration 3 the slowdown in flame speed and the reduced acceleration as the flame front travels the relatively longer distance between the second baffle plate and the square obstacle.

It is very interesting to note that using two baffles plates with a solid square obstacle having same blockage capacity in all the configurations, the recorded and predicted pressure is maximum in case of configuration 1 and minimum in case of configuration 3. It is also evident from Fig. 3 that, the flame exits chamber quickly in configuration 3 than in configuration 1. However, configuration 2 is in between the other two configurations in case of maximum overpressure and flame arrival time in the chamber. In case of configuration 1, though the flame has laminar nature until it reaches the first baffle plates at S2, quickly turn out to be highly wrinkled and turbulent due to jetting and contortion of the flame through the repeated obstacles. In this configuration the turbulent fluctuations are found to be progressively increasing and reach a maximum of $9 \mathrm{~m} / \mathrm{s}$ at $11.5 \mathrm{~ms}$. The laminar nature of flame front during the initial stages i.e up to $8 \mathrm{~ms}$ has caused longer blow down time from the chamber at later stages. It should be noteworthy, that the baffles and square obstacle in configuration 1 are almost all evenly spaced from bottom of ignition centre. While in configuration 3, flame found to be highly turbulent during initial stages followed by a faster decay at later stage. 
The second configuration has two baffles at S1, near to ignition centre and S3, away from the ignition centre and closed to the solid obstacle. It is noticed that, once the flame is distorted after reaching first baffle, flame front is slightly wrinkled with a higher surface area. However, re-laminarisation (reduction in speed and turbulence levels) of the flame between S1 and S3 took place and has caused to approach the square obstacle at a later stage compared to configuration 3 . It is evident from the computed RMS fluctuations at $10 \mathrm{~ms}$ as 5 and $8 \mathrm{~m} / \mathrm{s}$ and at $11.5 \mathrm{~ms}$ as 5 and $7 \mathrm{~m} / \mathrm{s}$ respectively in configuration 2 and 3 . This can be also observed by comparing the flame structure and its position between 10 and 11.5 ms from Fig. 3 (b) and (c). Similarly, from configuration 3 it is noticed that, due to succession of baffles close to the ignition centre at S1 and S2, flame front is highly distorted and wrinkled before approaching square obstacle. However, this configuration has recorded lowest pressure due to sudden deceleration between S2 and solid square obstacle as shown in Fig. 6 (b). The flame deceleration after $\mathrm{S} 2$ is also confirmed by the experimental measurements.

As a confirmation of the changing flame structure due to the location of the baffle plates upstream of the square obstacle, Fig. 7 shows measured images of $\mathrm{OH}$ near the obstacle for configurations 1 and 3. Also shown in Fig. 7 are the computed reaction rates for these cases around the same region. Assuming the $\mathrm{OH}$ here gives an adequate representation of the flame front, it is clear that the flame is much more wrinkled in configuration 1 where the baffle plate is closer to the obstacle. This is also evident in the calculations and hence confirming that, the turbulence generated by the baffle plates decays rather quickly, so the relative position and sequencing of obstacles is another important factor in explosions. It can be concluded that, with reference to an observer at the exit of chamber, the loss/damage due to the explosion pressure, is relatively less when solid obstacles are close to ignition origin, compared to that of when obstacles are relatively at longer distance.

The results presented in this paper and the good agreement obtained between measurements and computations, confirm the applicability of the DFSD model in predicting the explosion generated overpressure history, maximum overpressure and other flame characteristics much more accurately that the simple FSD model used earlier by the same authors (Ibrahim, Malalasekera, Gubba, \& Masri, 2007). Arguably, this is substantiating the dynamic nature of the present model and its capability to capture unresolved contributions (Knikker et al., 2004) of the flame surface density.

\section{Conclusions}

Explosion deflagrating flames in three different flow configurations have been simulated using a novel dynamic flame surface density (DFSD) turbulent combustion model implemented in a compressible Large Eddy Simulation code. Primarily, the applicability of the model to explosions is examined by validating the generated pressure and other flame characteristics, such as flame structure, position, speed and acceleration against published data. The model has resulted in predictions of pressure and flame structures that are superior to those obtained earlier using a simple flame surface density (FSD) model.

It is found that the position of solid obstruction with respect to each others has a significant impact on the magnitude of the explosion pressure as well as the spatial flame structure. Large separation between the solid baffle plates allows sufficient 
residence time for turbulence decay causing flow re-laminarisation and hence lowering pressures with a much smooth flame fronts. 


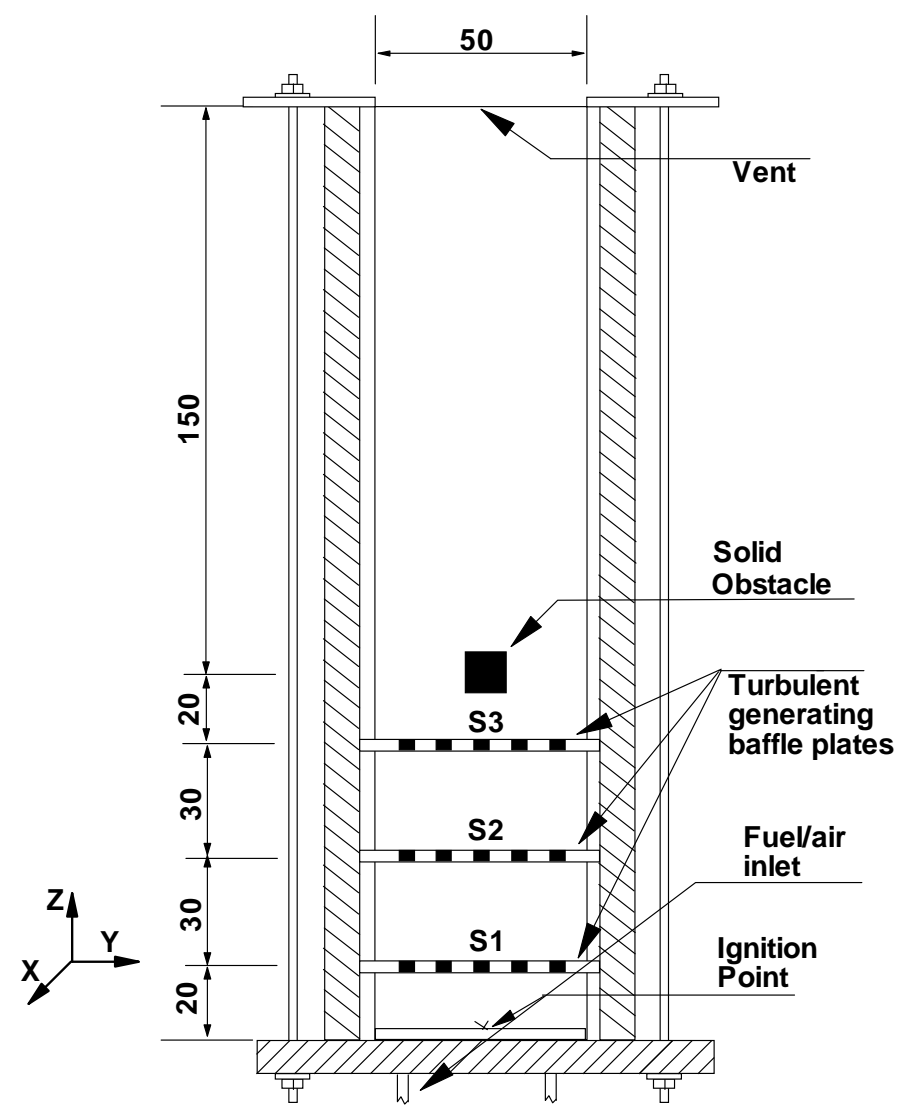

Fig. 1. Schematic of the experimental rig used for explosion of deflagrating flame. All dimensions are in $\mathrm{mm}$

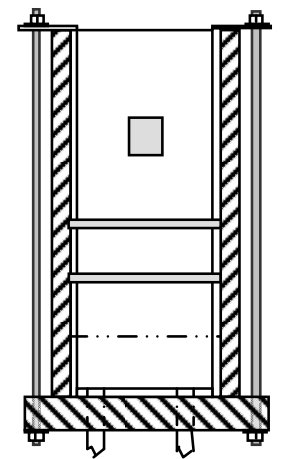

Configuration 1

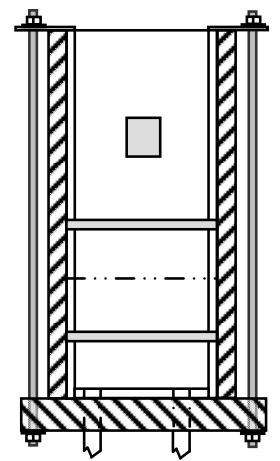

Configuration 2

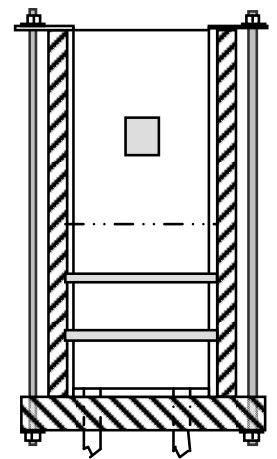

Configuration 3

Fig. 2. Various configurations employed in the present simulations. 


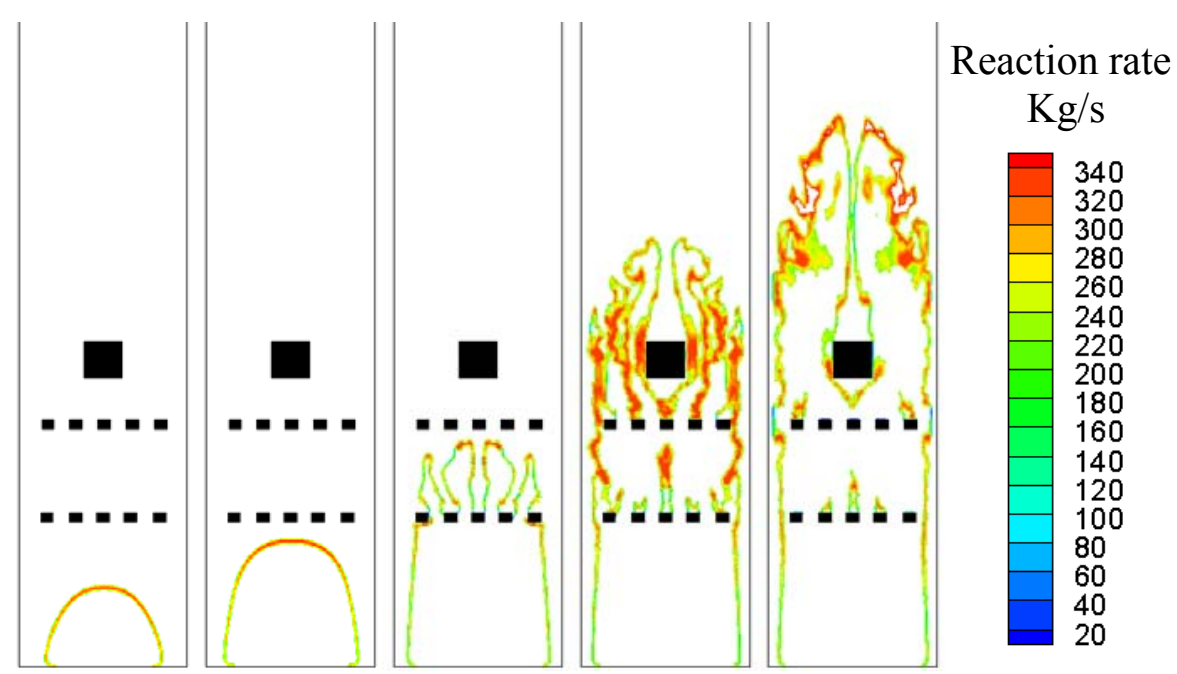

(a)

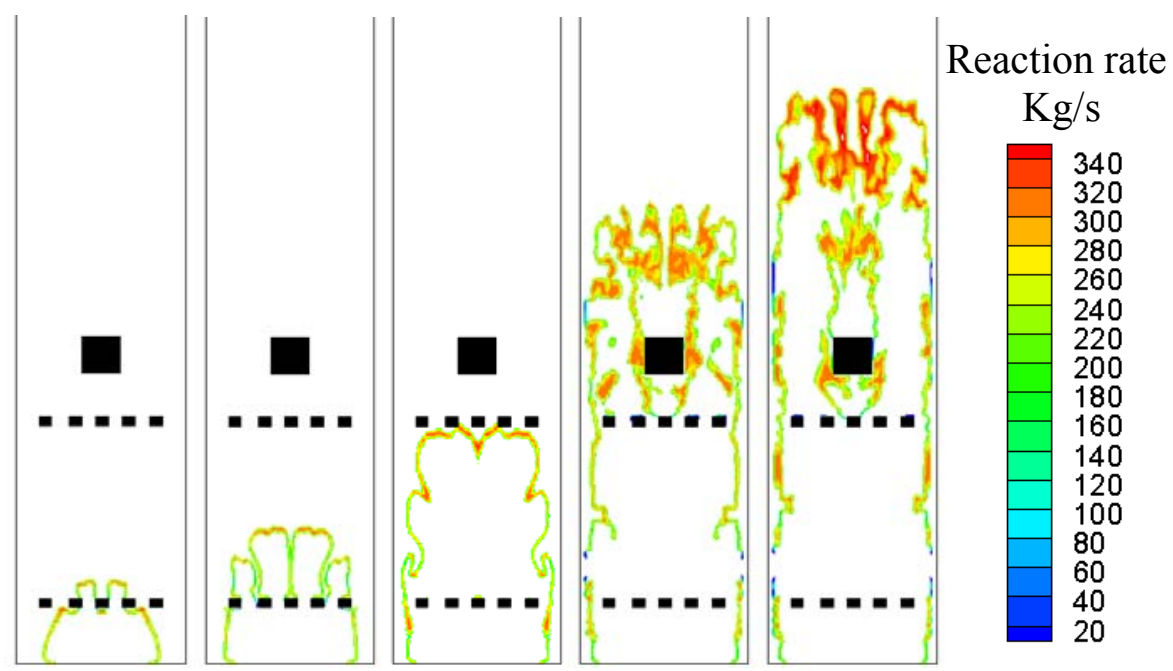

(b)

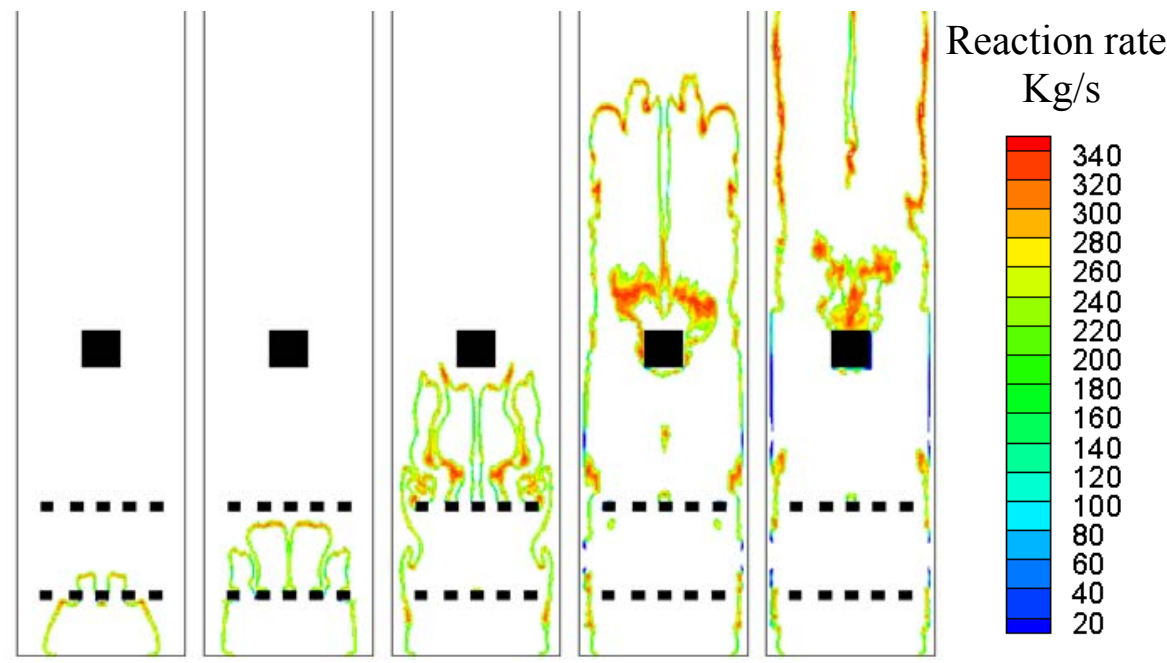

(c)

Fig. 3. Predicted flame structure in terms of reaction rate contour from three configurations at $6,8,10,11.5$ and $12.0 \mathrm{~ms}$ after ignition. (a) Configuration 1 (b) Configuration 2 (c) Configuration 3 


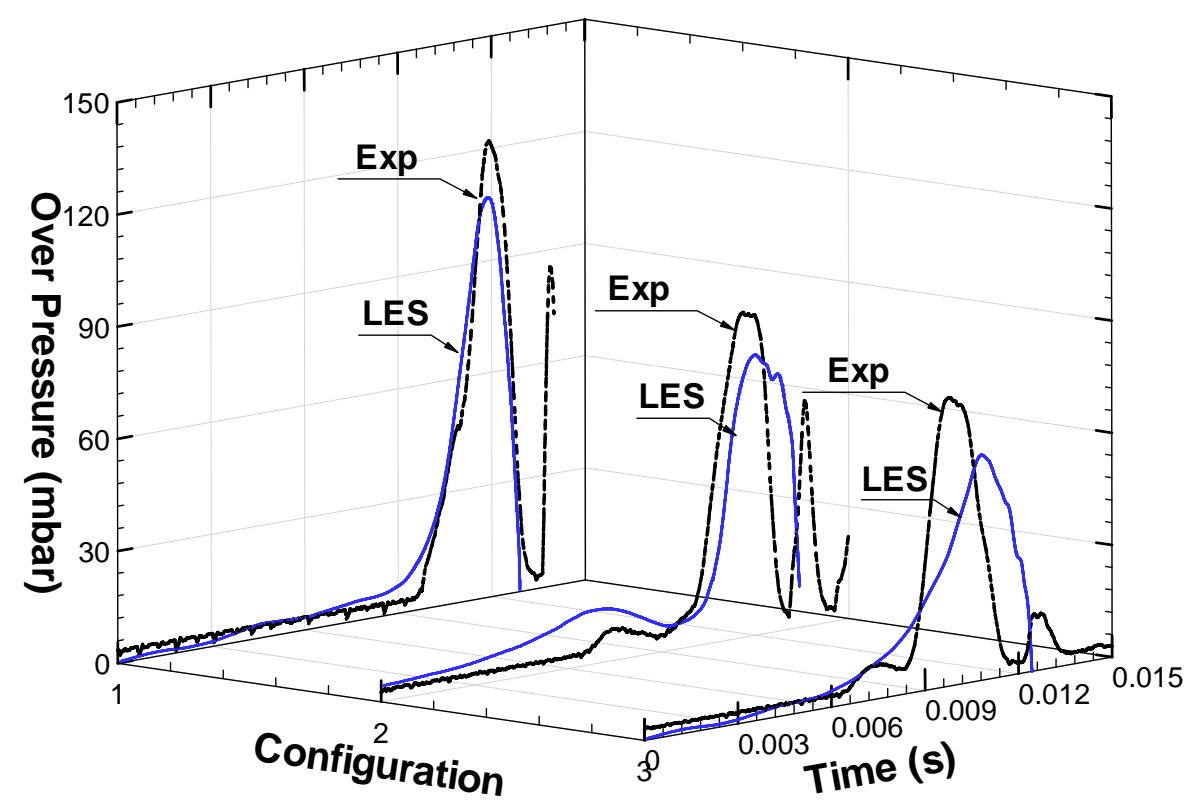

Fig. 4. Comparisons between predicted and measured pressure vs. time

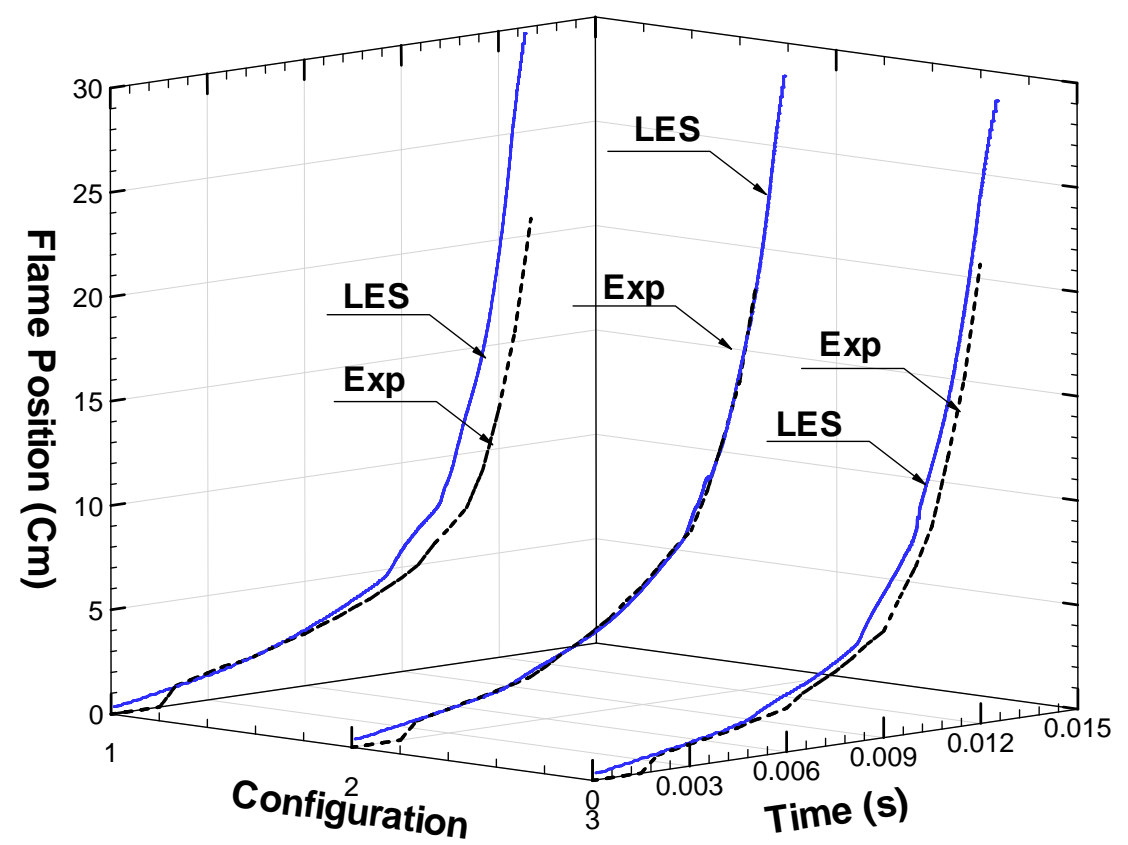

Fig. 5. Comparisons between predicted and measured flame position vs. time 


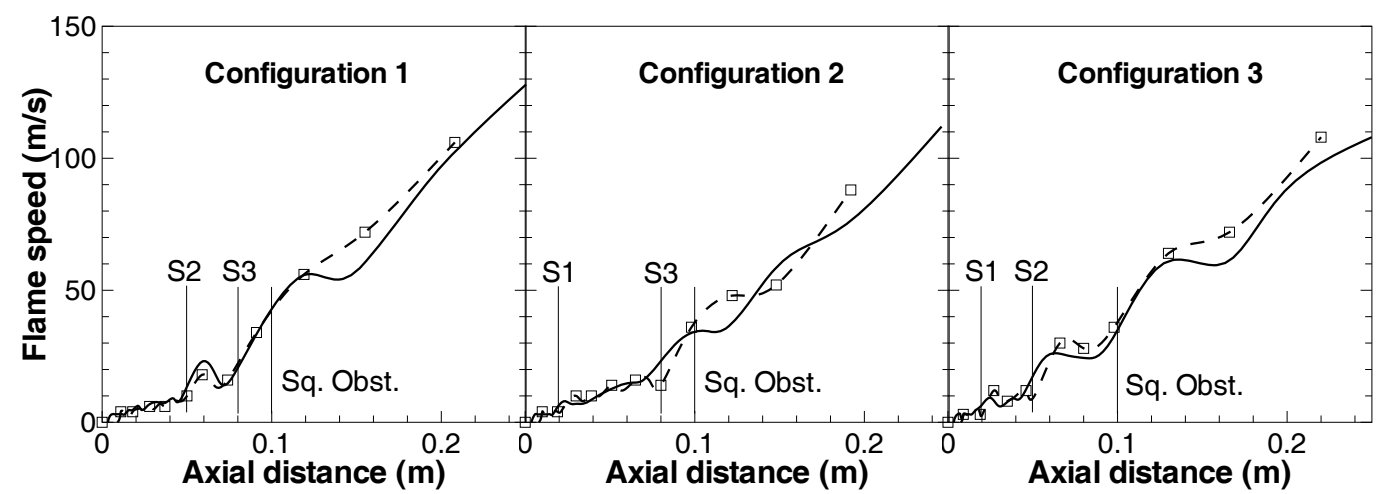

(a)

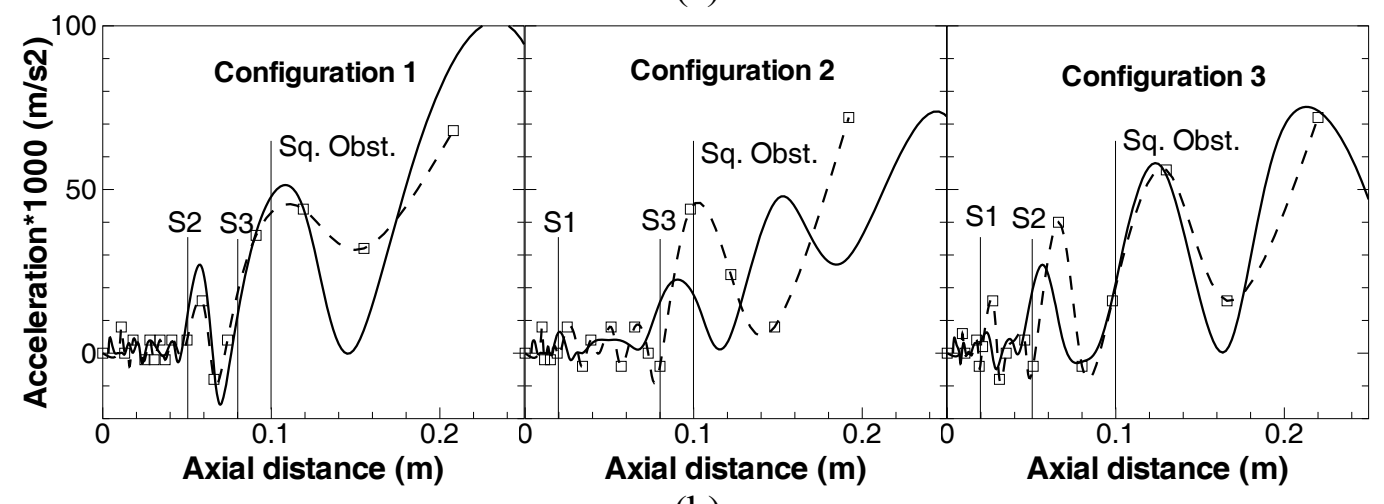

(b)

Fig. 6. Comparisons between predicted (Solid line) and measured (Dashed lines with square symbols) (a) flame speed (b) flame acceleration vs. axial distance. The location of baffle stations (S1, S2 and S3) and the square solid obstacle are shown.

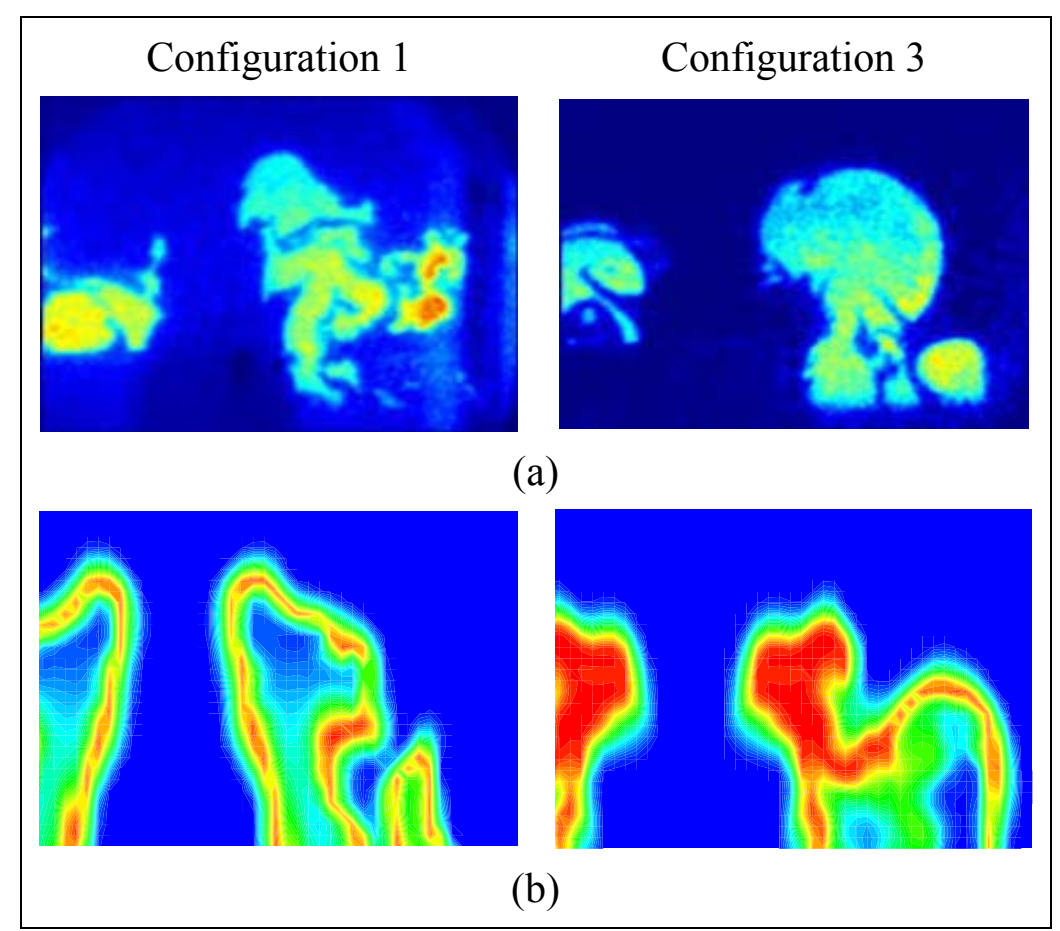

Fig.7. Comparison of $\mathrm{OH}$ images and corresponding flame fronts from LES simulations. (a) $\mathrm{OH}$ images (b) Reaction rate contours. 


\section{References}

Abdel-Gayed, R. G., Bradley, D., \& Lawes, M. (1987). Turbulent burning velocities: A general correlation in terms of straining rates. Proceedings of the Royal Society of London. Series A, Mathematical and Physical Sciences, 414, 389-413.

Bray, K. N. C. (1990). Studies of the turbulent burning velocity. Proceedings of the Royal Society: Mathematical and Physical Sciences, A431, 315.

Bray, K. N. C., Libby, P. A., \& Moss, J. B. (1985). Unified modeling approach for premixed turbulent combustion-Part I: General formulation. Combustion and Flame, 61(1), 87-102.

Chakravarthy, V. K., Smith, T. M., \& Menon, S. (1999). First International Symposium on Turbulence and Shear Flow Phenomena, Santa Barbara, CA. 181.

Germano, M., Piomelli, U., Moin, P., \& Cabot, W. H. (1991). A dynamic subgrid-scale eddy viscosity model. Physics of Fluids A: Fluid Dynamics, 3(7), 1760-1765.

Gubba, S. R., Ibrahim, S. S., Malalasekera, W., \& Masri, A. R. (2007). LES modelling of propagating turbulent premixed flames using dynamic flame surface density model. Paper presented at the $2^{\text {Nd }}$ ECCOMAS Thematic Conference on Computational Combustion, Delft University of Technology, The Netherlands.

Hawkes, E. R., \& Cant, R. S. (2001). Implications of a flame surface density approach to large eddy simulation of premixed turbulent combustion. Combustion and Flame, 126(3), 1617-1629.

Ibrahim, S. S., Hargrave, G. K., \& Williams, T. C. (2001). Experimental investigation of flame/solid interactions in turbulent premixed combustion. Experimental Thermal and Fluid Science, 24(3-4), 99-106.

Ibrahim, S. S., Malalasekera, W., Gubba, S. R., \& Masri, A. R. (2007). LES modelling of explosion propagating flame inside vented chambers with built-in solid obstructions. Paper presented at the $21^{\text {St }}$ International Colloquium on the Dynamics of Explosions and Reactive Systems, ENSEMA, Poitiers, France.

Ibrahim, S. S., \& Masri, A. R. (2001). The effects of obstructions on overpressure resulting from premixed flame deflagration. Journal of Loss Prevention in the Process Industries, 14(3), 213-221.

Kent, J. E., Masri, A. R., \& Starner, S. H. (2005). A new chamber to study premixed flame propagation past repeated obstacles. Paper presented at the $5^{\text {Th }}$ Asia-Pacific Conference on Combustion, The University of Adelaide, Adelaide, Australia.

Kirkpatrick, M. P., Armfield, S. W., Masri, A. R., \& Ibrahim, S. S. (2003). Large eddy simulation of a propagating turbulent premixed flame. Flow, Turbulence and Combustion, 70(1-4), 1-19. 
Knikker, R., Veynante, D., \& Meneveau, C. (2004). A dynamic flame surface density model for large eddy simulation of turbulent premixed combustion. Physics of Fluids, 16(11), L91-L94.

Leonard, B. P. (1979). A stable and accurate convective modelling procedure based on quadratic upstream interpolation. Computer Methods in Applied Mechanics and Engineering, 19, 59-98.

Leonard, B. P. (1987). SHARP simulation of discontinuities in highly convective steady flow (Technical memorandum No. NASA-TM-100240)

Moin, P., Squires, K., Cabot, W., \& Lee, S. (1991). A dynamic subgrid-scale model for compressible turbulence and scalar transport. Physics of Fluids A: Fluid Dynamics, 3(11), 2746-2757.

Patel, S., Ibrahim, S. S., Yehia, M. A., \& Hargrave, G. K. (2003). Investigation of premixed turbulent combustion in a semi-confined explosion chamber. Experimental Thermal and Fluid Science, 27(4), 355-361.

Patel, S., Jarvis, S., Ibrahim, S. S., \& Hargrave, G. K. (2002). An experimental and numerical investigation of premixed flame deflagration in a semiconfined explosion chamber. Proceedings of the Combustion Institute, 29(2), 1849-1854.

Smagorinsky, J. (1963). General circulation experiments with the primitive equations. Monthly Weather Review, 91(3), 99-164.

Veynante, D., \& Poinsot, T. (1997). Large eddy simulations of the combustion instabilities in turbulent premixed burners. Centre for Turbulence Research Annual Research Briefs, 253-275.

Werner, H., \& Wengle, H. (1991). Large-eddy simulation of turbulent flow over and around a cube in a plate channel. $8^{\text {Th }}$ Symposium on Turbulent Shear Flows, Munich, Germany. 